\section{Inaccuracy of FHSA registers: help from electoral registers}

\author{
Graham Bickler, Stephen Sutton
}

South East London Health Authority, London SE1 9RY

Graham Bickler, consultant in public health medicine

\section{ICRF Health Behaviour} Unit, Institute of Psychiatry, London SE5 8AF

Stephen Sutton, senior scientist

Correspondence to: Dr Bickler.

$B M \mathcal{F}$ 1993;306: 1167 electoral register. ${ }^{4}$

\section{Methods and results}

The inaccuracy of family health services authority (FHSA) registers has important implications for the breast and cervical screening programmes. ${ }^{1-3}$ For example, $35 \%$ of breast screening invitations in south east London did not reach the women they were intended for. ${ }^{1}$ Although the problem is widely recognised, there has been little discussion on how the registers can be used more effectively, though one approach is to use them in combination with the

In the context of a research programme on uptake of breast screening, we checked screening batch lists from Lambeth, Southwark, and Lewisham FHSAs for women aged 50-64 against the relevant electoral registers. The women were divided into "matches" and "non-matches." An interviewer went to random samples of the addresses listed to establish whether the woman lived there and if so to obtain information on sociodemographic characteristics and health beliefs. This visit was preceded by a letter and the number of letters returned undelivered by the Post Office (Post Office returns) was recorded.

The table shows that an estimated $73.3 \%$ of women on the FHSA lists were living at the address listed. This is a measure of the accuracy of the FHSA register as assessed by comparison with information obtained by visiting the address. The "matches" column shows that of those women whose names appeared on both registers, $91.9 \%$ were living at the address listed. Thus, using the two registers in combination yielded a much better "hit rate." By contrast, only $12.7 \%$ of the non-matches were residing at the address listed. Analysis of the interview data did not show any substantive differences between the matches and non-matches.

Checking the FHSA register (for women age 50-64) against the electoral register and targeting only those whose names appeared on both registers raised the accuracy of the addresses obtained from $73.3 \%$ to $91.9 \%$. Only $4 \cdot 1 \%$ of those actually living at the FHSA register address would be missed, but $23.5 \%$ of the original addresses would not need to be visited. No significant biases were introduced. The number of Post Office returns underestimated the number of wrong addresses by $66 \cdot 1 \%$.

\section{Comment}

Given the simplicity and benefits of checking one register against another, what are the implications? For population based research-particularly where it involves personal interviews - such a checking procedure would increase efficiency. Costs would decrease as interviewers would go to fewer addresses to obtain the same sample size. Put another way, it would be possible to have larger sample sizes for the same cost without introducing important biases.

For the breast and cervical screening programmes which rely on the FHSA register, crude uptake rates could be adjusted by checking a sample of the FHSA register against the electoral register, making assumptions about the probabilities of matches and nonmatches actually living at the addresses listed, and altering the denominator appropriately. A correction could also be done by making assumptions about the relation between the Post Office return rate and the true inaccuracy rate: it seems from this and other studies that a ratio of between $2: 1$ and $3: 1$ is reasonable. ${ }^{15}$ This would be far cheaper than checking against the electoral register, but less accurate. FHSA lists could be updated more efficiently by asking general practitioners and their practice staff to focus on the non-matches, thus lightening their administrative load.

We recommend that researchers consider using this technique to improve the efficiency of research that uses FHSA registers as a sampling frame, and that the national breast screening programme use this technique -along with the Post Office return rate-to provide more valid information for comparing uptake rates.

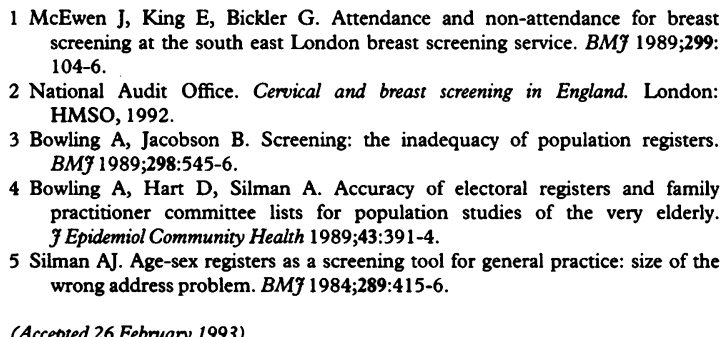

Summary of information obtained from interviewers' visits to 1691 addresses on FHSA lists matching and not matching those on electoral register. Values are numbers (percentages)

\begin{tabular}{|c|c|c|c|}
\hline & Matches & Non-matches ${ }^{\star}$ & Full samplet \\
\hline $\begin{array}{l}\text { Original sample } \\
\text { Addresses visited }\end{array}$ & $977(75 \cdot 6)^{1293(76 \cdot 5)}$ & $189(47.5)^{398(23.5)}$ & $1691(100)$ \\
\hline $\begin{array}{l}\text { Outcome: } \\
\text { Address did not exist } \\
\text { Address visited but no information obtained } \\
\text { Target person alive and living at the address } \ddagger \\
\text { Target person not known at the address or had moved } \\
\text { Post Office returns } \int \\
\text { Other }\end{array}$ & $\begin{array}{r}0 \\
20(2 \cdot 0) \\
898(91 \cdot 9) \\
55(5 \cdot 6) \\
14(25 \cdot 5) \quad 4(0.4)\end{array}$ & 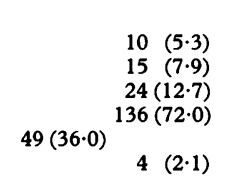 & $\begin{array}{r}21(1 \cdot 2) \\
58(3 \cdot 4) \\
1239(73 \cdot 3) \\
359(21 \cdot 2) \\
122(33.9) \quad \\
14(0.8)\end{array}$ \\
\hline Total & $977(100)$ & $189(100)$ & $1691(100)$ \\
\hline
\end{tabular}

*Screening batch address not found on electoral register, or address found but no one with same first or last name was listed.

†Estimates for full sample derived by combining frequencies for matches and non-matches using appropriate weights, rounding to nearest integer, and then converting to percentage.

converting to percentage. $100 \mathrm{a} /(\mathrm{a}+\mathrm{b})$, where $\mathrm{a}=(12 \cdot 7)(398)$ and $\mathrm{b}=(91.9)(1293)$.

$\oint$ Person not known at address or had moved. There were also five Post Office returns from 10 non-existent addresses among non-matches. 